\title{
TWO REMARKS ON RIEMANN SURFACES
}

\author{
J. M. RODRÍGUEZ*
}

\begin{abstract}
We study the relationship between linear isoperimetric inequalities and the existence of non-constant positive harmonic functions on Riemann surfaces.

We also study the relationship between growth conditions of length of spheres and the existence of Green's function on Riemann surfaces.
\end{abstract}

\section{Introduction}

The theme of this paper is to clarify some connections between different conformal invariants of Riemann surfaces.

By a Riemann surface $R$ we denote a two-dimensional surface with a complete metric of constant negative curvature -1 . In this case, the universal covering space of $R$ is the unit disk $\Delta ; R$ is endowed with its Poincaré metric, i.e., the metric obtained by projecting the Poincaré metric of the unit disk $d s=2\left(1-|z|^{2}\right)^{-1}|d z|$ onto $R$. The unit disk $\Delta$ is isometric to the upper-half plane $U$ endowed with its Poincaré metric $d s=|d z| / y, z=x+i y \in U$. We will use both models of the hyperbolic plane. The only Riemann surfaces which are left out are the sphere, the plane, the punctured plane and the tori.

We shall say that a Riemann surface $R$ satisfies a linear isoperimetric inequality (LII) if there exists a finite constant $h(R)$ so that for every bounded open set $G$ with smooth boundary we have

$$
A(G) \leq h(R) L(\partial G) .
$$

Here and from now on, $A, L$ and $d$ refer to Poincaré area, length and distance of $R$.

There are close connections between LII and some conformal invariants on Riemann surfaces, namely the bottom of the spectrum of the LaplaceBeltrami operator $b(R)$, and the exponent of convergence $\delta(R)$. These connections are described in the next two known results.

*Research supported in part by a grant of the DGICYT, Ministerio de Educación y Ciencia, Spain. 
Theorem A [Ch], [Bu, p. 228], [FR1]. A Riemann surface $R$ satisfies a linear isoperimetric inequality if and only if $b(R)>0$. In fact,

$$
\frac{1}{4} \leq b(R) h(R)^{2} \text { and } b(R) h(R)<\frac{3}{2} .
$$

The next result is a well known theorem of Elstrodt-Patterson-Sullivan:

Theorem B [S, p. 333]. A Riemann surface $R$ satisfies a linear isoperimetric inequality if and only if $\delta(R)<1$. In fact,

$$
b(R)= \begin{cases}\frac{1}{4}, & \text { if } 0 \leq \delta(R) \leq \frac{1}{2}, \\ \delta(R)(1-\delta(R)), & \text { if } \frac{1}{2} \leq \delta(R) \leq 1 .\end{cases}
$$

We shall be particularly interested in the class $\mathcal{B}$ of Riemann surfaces which do not satisfy a linear isoperimetric inequality.

A theorem of Myrberg [T, p. 522] states that if $\delta(R)<1$ (if $R$ satisfies a LII) then $R$ has a Green's function $\left(R \notin O_{G}\right.$ in the language of classification theory). If $R$ is a plane domain (in fact; if $R$ is a surface of almost finite genus [SN, p. 193]), the following conditions are equivalent [SN, p. 194]:

(i) $R$ has Green's function $\left(R \notin O_{G}\right)$,

(ii) $R$ has a non-constant positive harmonic function $\left(R \notin O_{H P}\right)$,

(iii) $R$ has a non-cosntant bounded harmonic function $\left(R \notin O_{H B}\right)$,

(iv) $R$ has a non-constant harmonic function with finite Dirichlet integral $\left(R \notin O_{H D}\right)$.

The Dirichlet integral of a function is the square of the $L^{2}$-norm of its gradient.

For arbitrary Riemann surfaces the following strict inclusions are well known:

$$
O_{G} \subset O_{H P} \subset O_{H B} \subset O_{H D} .
$$

One would like to understand exactly how the class $\mathcal{B}$ fits into this chain.

As we have said above, in the case of surfaces of almost finite genus, $O_{G}=O_{H P}=O_{H B}=O_{H D} \subset \mathcal{B}$. The inclusion is strict, even in the planar case, as it is shown by the example $R_{0}=\Delta \backslash\left(\cup_{k=1}^{\infty}\left\{2^{-k}\right\} \cup\{0\}\right)$ : $R_{0} \notin O_{H D}$ because it is a plane domain whose boundary has positive logarithmic capacity [T, p. 81]; $R_{0} \in \mathcal{B}$ because $\cup_{k=1}^{\infty}\left\{2^{-k}\right\} \cup\{0\}$ is a discrete set with an accumulation point in $\Delta$ [FR1, Theorem 4]. This example shows that $\mathcal{B}$ is not contained in $O_{H P}, O_{H B}$ or $O_{H D}$. 
The inclusion $O_{H D} \subset \mathcal{B}$ is not true in general. To describe why, consider the real linear space $H D(R)$ of harmonic functions in $R$ with finite Dirichlet integral. Then, we have the following

Theorem $\mathbf{C}[\mathbf{R o}]$. Let $R$ be a Riemann surface which satisfies a linear isoperimetric inequality. If there exists in $R$ a set of disjoint simple closed curves $\left\{\gamma_{j}\right\}_{j=1}^{m}$, such that $R \backslash \cup_{j} \gamma_{j}$ contains n connected components of infinite area, then

$$
\operatorname{dim} H D(R) \geq n .
$$

This inequality is best possible: for each $n \geq 1$, there exists a Riemann surface $R_{n}$ satisfying the hypothesis of the theorem and such that $\operatorname{dim} H D\left(R_{n}\right)=n$.

In particular, for $n=1$ we have the following

Corollary. There is a Riemann surface $R_{1}$ with LII and such that every harmonic function with finite Dirichlet integral on $R_{1}$ is constant.

$R_{1}$ can be constructed verifying the extra customary hypothesis of bounded geometry $[\mathbf{K}]$, which in our context simply means that the injectivity radius $\iota\left(R_{1}\right)$ is positive. For any Riemann surface $R, \iota(R)$ is defined as

$$
\iota(R)=\inf \{\iota(p): p \in R\},
$$

where $\iota(p)$ is the injectivity radius of the geodesic exponential map centered at $p$.

Summarizing, the situation is the following:

$$
O_{G} \subset \mathcal{B}, \quad O_{G} \subset O_{H P} \subset O_{H B} \subset O_{H D},
$$

and there are no inclusion relationships between $\mathcal{B}$ and $O_{H D}$. But, is there any inclusion relationship between $\mathcal{B}$ and $O_{H B}$ or between $\mathcal{B}$ and $O_{H P}$ ?

It was natural to expect the inclusion $O_{H B} \subset \mathcal{B}$ to be true. For instance, if $R$ is a surface of almost finite genus, then $O_{H B}=O_{G} \subset \mathcal{B}$; if $R$ has a LII and is a regular covering of a compact surface (then $R$ has infinite genus), one can prove that the Varopoulos's index $\alpha(R)$ [V3] is positive, and this implies the existence of non-constant bounded harmonic functions. Then, the inclusion is true for finite genus and for two extremal cases with infinite genus: almost finite genus and covering spaces of compact surfaces.

In this paper we prove by an example that the inclusion $O_{H B} \subset \mathcal{B}$ is not true. In fact, we prove that the weaker inclusion $O_{H P} \subset \mathcal{B}$ is not true, even with the hypothesis $\iota>0$ : 
Theorem 1. There exists a Riemann surface $\mathcal{M}$ with $L I I, \iota(\mathcal{M})>0$, and such that every positive harmonic function on $\mathcal{M}$ is constant.

A most interesting example in the classification of Riemann surfaces was obtained by Lyons [L]. He constructed two quasiisometric (and, in particular, quasiconformaly equivalent) Riemann surfaces, one of them belonging to $O_{H P}$ while the other supports non-constant bounded harmonic functions. We shall be using his construction as a basic ingredient in the proof of theorem 1.

The relationship between growth conditions of area of balls and the existence of Green's function is a well-known and classical issue. We refer, for instance, to $[\mathbf{D}],[\mathbf{E}],[\mathbf{F}],[\mathbf{F R 2}],[\mathbf{G}],[\mathbf{K a}],[\mathbf{L S}],[\mathbf{V} \mathbf{1}]$ and the references therein for some general (in arbitrary Riemannian manifolds) geometric and topological conditions related to the existence of Green's function. Green's function exists if and only if there exists a positive nonconstant superharmonic function or equivalently if Brownian motion on the surface is transient (see, e.g. [AS, p. 204], [V1]).

If $p$ is a point of a Riemann surface $R$ and $t$ is a positive number we denote by $A_{R}(p, t)$ and $L_{R}(p, t)$, the area of $B(p, t)$ and the length of $\partial B(p, t)$ respectively, where $B(p, t)$ is the ball of radius $t$ centered at $p$. Of course, $A_{R}(p, t) \leq A_{\Delta}(0, t)=4 \pi \sinh ^{2}(t / 2) \approx \pi e^{t}$ as $t \rightarrow \infty$, and $L_{R}(p, t) \leq L_{\Delta}(0, t)=2 \pi \sinh t \approx \pi e^{t}$ as $t \rightarrow \infty$.

The following theorem is known.

Theorem D. (i) If for a point $p_{0} \in R$ and constants $c_{0}, t_{0}$

$$
A_{R}\left(p_{0}, t\right) \geq c_{0} e^{t}, \text { for every } t \geq t_{0},
$$

then $R$ has a Green's function.

(ii) Given a function $\psi:(0, \infty) \rightarrow(0, \infty)$, increasing, and such that

$$
\lim _{t \rightarrow \infty} \frac{\psi(t)}{e^{t}}=0
$$

there exists a Riemann surface $R$ and a point $p_{0} \in R$ so that

$$
A_{R}\left(p_{0}, t\right) \geq \psi(t), \text { for every } t \geq t_{1},
$$

but $R$ has no Green's function.

Part (i) is elementary and part (ii) is due to Nicholls [N]. We do remark that in the example of (ii) of theorem $\mathrm{D}, R$ can be chosen to be planar. 
It has been suggested by N. Varopoulos that in our situation, i.e., constant negative curvature, a uniform exponential growth of the area should imply that Green's function exists. More precisely, if there are positive constants $c, \alpha, t_{0}$, such that

$$
A_{R}(p, t) \geq c e^{\alpha t} \text {, for every } t \geq t_{0}, p \in R,
$$

can we deduce that $R$ has a Green's function?

The following theorem [FR2] answers the question.

Theorem E. (i) If $R$ is a non-compact Riemann surface of finite genus and there are positive constants $c, t_{0}$, such that

$$
A_{R}\left(p, t_{0}\right) \geq c, \text { for every } p \in R,
$$

then $R$ has a Green's function.

(ii) There exists a Riemann surface $R$ so that

$$
A_{R}(p, t) \geq c e^{\alpha_{0} t}, \text { for every } t \geq t_{0}, p \in R,
$$

where $c, \alpha_{0}, t_{0}$ are positive numbers, and such that $R$ does not have a Green's function.

Varopoulos in [V2, p. 271] gives an example like the one in theorem $\mathrm{E}$ with $\alpha_{0}=1 / 2$, but with variable curvature. Notice that topologically his example is the plane.

The example constructed in [FR2] has exponential growth of area of balls but the length of spheres does not grow. As a matter of fact, there exists a point $p$ and positive numbers $t_{n}$ converging to infinity, such that $L\left(p, t_{n}\right)$ remain bounded as $n \rightarrow \infty$. And actually that is why the Riemann surface has no Green's function.

Therefore, it is natural to ask if the stronger condition

$$
L_{R}(p, t) \geq c e^{\alpha t}, \text { for every } t \geq t_{0}, p \in R,
$$

implies that $R$ has a Green's function. Observe that this implies

$$
A_{R}(p, t) \geq c_{\varepsilon} e^{\alpha t}, \text { for every } t \geq t_{0}+\varepsilon, p \in R, \varepsilon>0,
$$

because $A_{R}(p, t)=\int_{0}^{t} L_{R}(p, s) d s$.

Observe that if $L_{R}\left(p_{0}, t\right) \geq c e^{t}$, for some point $p_{0} \in R$ and for every $t \geq t_{0}$ then, of course, $R$ has a Green's function, while, on the other hand, a classical criterion of Ahlfors $[\mathbf{A}]$ says that if $R$ has a Green's function then $L_{R}\left(p_{0}, t\right)$, grows rapidly; in fact,

$$
\int^{\infty} \frac{d t}{L_{R}\left(p_{0}, t\right)}<\infty
$$

Here we prove 
Theorem 2. There exists a Riemann surface $\mathcal{N}$ so that

$$
L_{\mathcal{N}}(p, t) \geq c e^{t / 2}, \text { for every } t \geq t_{0}, p \in \mathcal{N},
$$

where $c, t_{0}$ are positive numbers, and such that $\mathcal{N}$ does not have a Green's function.

Notice in particular that theorem 2 has theorem E, (ii) as an immediate corollary. The proof of theorem 2 is simpler and gives the precise rate $\alpha_{0}=1 / 2$. We do not know what the sharp rate $\alpha_{0}$ in theorem $\mathrm{E}$ is.

The organization of the paper is as follows. In Section 2 we prove theorem 1. In Section 3 we construct the Riemann surface $\mathcal{N}$ of theorem 2, while in Section 4 we prove that if satisfies the inequality $L_{\mathcal{N}}(p, t) \geq$ $c e^{t / 2}$. In Section 5 we prove that $\mathcal{N}$ has no Green's function.

\section{Proof of Theorem 1}

The desired Riemann surface $\mathcal{M}$ will be obtained with the help of two graphs $G$ and $G_{0}$.

In the set of vertices of any connected graph we can define a natural distance:

$$
d(p, q)=\inf \{\text { length of the paths from } p \text { to } q\},
$$

where all edges have length 1 . This will be "the distance" in all graphs of this section. The degree of a vertex is the number of its neighbours, i.e., the number of vertices at distance 1 from it.

The vertices of the graphs $G$ and $G_{0}$ are simply the elements of the free group on two generators $\Gamma=\langle a, b\rangle$. Now we define the edges of $G$ and $G_{0}$.

Each element of $\Gamma$ can be expressed as a word with the letters $a, a^{-1}$, $b, b^{-1}$. This expression is unique if no simplification of contiguous letters is possible; in this case we say that the word is a reduced word.

For each $n$, we choose $\theta_{n}$ a length preserving permutation of the words of length greater or equal than $n$. This permutation must have order $3^{n-1}$ and must satisfy that $g$ and $g \theta_{n}$ start on the left with the same letter for all reduced words $g$ of length $n$. If $g$ is a reduced word of length at least $n$, there is a unique factorization $g=g_{1} g_{2}$ with length $\left(g_{1}\right)+$ length $\left(g_{2}\right)=$ length $(g)$, and length $\left(g_{2}\right)=n$. We also require that $\theta_{n}$ satisfies $g \theta_{n}=g_{1}\left(g_{2} \theta_{n}\right)$ (observe that length $\left(g \theta_{n}\right)=$ length $(g)$, because $\theta_{n}$ does not change the leftmost element of $\left.g_{2}\right)$. 
The sequence $\left\{\theta_{n}\right\}_{n=1}^{\infty}$ can be arranged so that it verifies the recursion

$$
\begin{aligned}
\left(\theta_{n}\right)^{3} & =\theta_{n-1} \\
\theta_{1} & =\text { identity }
\end{aligned}
$$

Let $\left\{r_{n}\right\}$ and $\left\{s_{n}\right\}$ be two sequences of natural numbers satisfying $n<r_{n}<s_{n}<r_{n+1}$ and $s_{n}-r_{n}>\left(3^{n-1}\right)^{2+\varepsilon}$, for some positive $\varepsilon$ and for each $n$.

We define the set of edges of $G$ in the following way. If $g_{1}$ and $g_{2}$ are reduced words of $\Gamma$, there is an edge between them if and only if they verify one of the conditions:

(i) $g_{1} g_{2}^{-1}=a^{ \pm 1}$,

(ii) $g_{1} g_{2}^{-1}=b^{ \pm 1}$,

(iii) $g_{1}=g_{2} \theta_{n}^{ \pm 1}$ and length $\left(g_{1}\right) \in\left[r_{n}, s_{n}\right]$ for some $n$.

Consequently, every vertex of $G$ has degree four or six.

$G_{0}$ is simply the Cayley graph of the group $\Gamma$, i.e., $g_{1}$ and $g_{2}$ are connected by an edge of $G_{0}$ only if (i) or (ii) is satisfied.

Then, every vertex of $G_{0}$ has degree four.

A way to build up our Riemann surface $\mathcal{M}$, modelled upon the graph $G$, is to use the so called Löbell $Y$-pieces, which are a standard tool for constructing Riemann surfaces. A clear description of these $Y$-pieces and their use is given in [C, Chapter X.3]. (In [L] there is an equivalent way of constructing Riemann surfaces.)

A Löbell $Y$-piece is a three-holed sphere, endowed with a metric of constant negative curvature -1 , so that the boundary curves are geodesics. The $Y$-pieces are a flexible tool: given any three positive numbers $a, b$, $c$, there is a $Y$-piece with boundary curves which have lengths $a, b, c$ (see [C, p. 248] and [Fe, p. 99] for details).

A $X$-piece (*-piece) is a four-holed (six-holed) sphere, endowed with a metric of curvature -1 , so that the boundary curves are geodesics. We can construct these pieces, for example, joining two (four) $Y$-pieces, by identifying corresponding boundary curves of the same length, in such a way that the resulting surface has genus zero.

We are free to choose the lengths of the four (six) boundary curves of the $X$-piece (*-piece). We choose the lengths of the boundary curves of a $X$-piece and a $*$-piece following the construction of the manifold $M$ in [L, p. 57] (a large "cylinder" in $M$ corresponds to a short boundary curve of our Riemann surface $\mathcal{M}[\mathbf{R}])$. The four boundary geodesics of a $X$-piece have the same length $\alpha$. Four of the boundary geodesics of a $*$-piece also have length $\alpha$; the order two boundary curves have the 
same length $\beta$, with $\beta \gg \alpha$. We take now infinitely many copies of both pieces.

We now build $\mathcal{M}$ by joining these pieces following the combinatorial design of $G$, with the $X$-pieces (*-pieces) in the place of the vertices of degree four (six), glueing boundary curves of the same length. $\mathcal{M}$ is a complete surface of constant negative curvature -1 , and since we have used only two distinct pieces to build up $\mathcal{M}$, it is obvious that $\iota(\mathcal{M})>0$.

The results of $[\mathbf{L}]$ give that every positive harmonic function on $\mathcal{M}$ is constant.

To prove that $\mathcal{M}$ has a LII we need to make precise the metric relationship between $G$ and $\mathcal{M}$. Following Kanai's terminology $[\mathbf{K}]$, we say that a mapping $\varphi$, not necessarily continuous, between two metric spaces

$$
\varphi:\left(M_{1}, d_{1}\right) \rightarrow\left(M_{2}, d_{2}\right)
$$

is a rough isometry if the following two conditions are satisfied:

(i) There are constants $a \geq 1$ and $b \geq 0$ such that

$$
a^{-1} d_{1}(x, y)-b \leq d_{2}(\varphi(x), \varphi(y)) \leq a d_{1}(x, y)+b,
$$

for all $x, y \in M_{1}$.

(ii) For some $\varepsilon>0$, the $\varepsilon$-neighbourhood of $\varphi\left(M_{1}\right)$ covers $M_{2}$.

A metric space $M_{1}$ is said to be roughly isometric to a metric space $M_{2}$ if there exists a rough isometry from $M_{1}$ into $M_{2}$. It can be checked that being roughly isometric is an equivalence relation between metric spaces.

It is obvious that the graph $G$ and the surface $\mathcal{M}$ are roughly isometric.

If $F$ is a graph, and $P$ is a subset of vertices of $F$ we define its boundary $\partial P$ by

$$
\partial P \equiv\{v \in V(F): d(v, P)=1\} .
$$

If $|\cdot|$ denotes the number of elements of a subset of vertices, the linear isoperimetric constant of $F$ is defined by

$$
h(F)=\sup _{P} \frac{|P|}{|\partial P|},
$$

where $P$ ranges over all the non-empty finite subsets of vertices of $F$.

Lemma K. Let $R$ be a Riemannian manifold with bounded geometry and let $F$ be a graph with bounded degree. If $R$ and $F$ are roughly isometric, then $R$ satisfies a linear isoperimetric inequality if and only if $F$ satisfies a linear isoperimetric inequality. 
To prove this lemma it is enough to combine two lemmas of Kanai [K, p. 401] (observe that the hypothesis of bounded geometry is satisfied in our case, and also that $m=\infty$ is allowed in the notation of Kanai).

Lemma $\mathrm{K}$ says that the surface $\mathcal{M}$ and the graph $G$ simultaneously verify or not a LII. Moreover, the definition of the linear isoperimetric constant in a graph implies that $G$ has a LII if the tree $G_{0}$ has a LII, because both have the same vertices and $G$ has more edges.

It is elementary that a regular tree of degree $d \geq 3$, satisfies the LII with constant $1 /(d-2)$. In particular, $G_{0}$ satisfies the LII

$$
|P| \leq \frac{1}{2}|\partial P|
$$

Lemma $\mathrm{K}$ then gives that our surface $\mathcal{M}$ satisfies LII. The proof of theorem 1 is complete.

\section{Constructing the Riemann surface $\mathcal{N}$}

Let $U$ be the upper-half plane $U=\{x+i y \in \mathbf{C}: y>0\}$ endowed with its Poincare metric $d s=|d z| / y$. Let $V_{0}$ be the closed subset of $\{x+i y:-1 \leq x \leq 1\}$ limited by the geodesic arcs $g_{1}, g_{2}, g_{3}$ :

$$
\begin{aligned}
& g_{1}=\{1+i y: y \geq 1\} \\
& g_{2}=\{-1+i y: y \geq 1\} \\
& g_{3}=\left\{x+i y: x^{2}+y^{2}=2,-1 \leq x \leq 1\right\}
\end{aligned}
$$

We define $V_{k}=T^{k} V_{0}$, for all integer $k$, where $T^{k}(z)=z+2 k$, and let $W_{1}$ be the closed periodic set $W_{1}=\cup_{k} V_{k}$. The boundary of $W_{1}$ is the union of the geodesic arcs $G_{1}^{k}=T^{k}\left(g_{3}\right)$. Observe that two geodesics $G_{1}^{k}$ and $G_{1}^{k+1}$ meets at the point $2 k+1+i$ with angle $\pi / 2$. We denote $\gamma_{1}^{k}=G_{1}^{2 k}$ and $\eta_{1}^{k}=G_{1}^{2 k+1}$.

Let $W_{2}$ be another copy of $W_{1}$. We denote by $G_{2}^{k}, \gamma_{2}^{k}, \eta_{2}^{k}$, the analogues in $W_{2}$ of $G_{1}^{k}, \gamma_{1}^{k}, \eta_{1}^{k}$, respectively.

If we join both copies $W_{1}$ and $W_{2}$ identifying $\eta_{1}^{k}$ with $\eta_{2}^{k}$, we obtain a Riemann surface $W$ with boundary. Let $\gamma_{k}$ be the simple closed geodesics in $W, \gamma_{k}=\gamma_{1}^{k} \cup \gamma_{2}^{k}$; then the boundary curves of $W$ are $\left\{\gamma_{k}\right\}_{k=-\infty}^{\infty}$.

Let $W^{\prime}$ be another copy of $W$. We denote by $\gamma_{k}^{\prime}$ the analogues in $W^{\prime}$ of $\gamma_{k}$. Finally, if we join $W$ and $W^{\prime}$ identifying $\gamma_{k}$ with $\gamma_{k}^{\prime}$, we obtain the complete Riemann surface $\mathcal{N}$. 


\section{Proof of the growth inequality of $\mathcal{N}$}

We need the following lemma.

Lemma. If $H_{0}=\{x+i y: y \geq \sqrt{2}\}$ and $H=\{x+i y: y \geq 1\}$, then

$$
L\left(\partial B(z, t) \cap H_{0}\right) \geq 2 \sinh t \arctan \left(\frac{\sqrt{2 \sqrt{2} \cosh t-3}}{\sqrt{2} \cosh t-1}\right),
$$

for all $z$ in $H$, and all radius $t>\log \sqrt{2}$.

Proof of the lemma:

Without loss of generality we can assume that $z=i$, because this point minimize $L\left(\partial B(z, t) \cap H_{0}\right)$, for every $t>\log \sqrt{2}(\log \sqrt{2}$ is the distance between $i$ and $i \sqrt{2}$ ). Let us fix a radius $t>\log \sqrt{2}$.

There are two points in $\partial B(i, t)$ with imaginary part $\sqrt{2}: u+i \sqrt{2}$ and $-u+i \sqrt{2}$. If we denote by $d$ the Poincaré distance in $U$, we have ([B, p. 130], [Fe, p. 38])

$$
\cosh d(z, w)=\frac{(\operatorname{Re} z-\operatorname{Re} w)^{2}+(\operatorname{Im} z)^{2}+(\operatorname{Im} w)^{2}}{2 \operatorname{Im} z \operatorname{Im} w} .
$$

In our case, $d(i, u+i \sqrt{2})=t$, and then

$$
\cosh t=\frac{u^{2}+3}{2 \sqrt{2}} .
$$

The length of $\partial B(i, t)$ is $2 \pi \sinh t$ [B, p. 132]. Let $a$ be the angle in $i$ between the geodesic arc which joins $i$ and $u+i \sqrt{2}$, and the geodesic $\{i y: y \geq 1\}$. Therefore,

$$
L\left(\partial B(i, t) \cap H_{0}\right) \geq 2 a \sinh t .
$$

The proof is finished, once we show that

$$
\tan a=\frac{\sqrt{2 \sqrt{2} \cosh t-3}}{\sqrt{2} \cosh t-1} .
$$

To see this, consider the geodesic $g$ which joins $i$ and $u+i \sqrt{2}$. The Poincaré geodesics in $U$ are the vertical straight lines and the circles orthogonal to the real axis. This implies that $g$ has the equation

$$
x^{2}-2 x_{0} x+y^{2}=1,
$$


with $x_{0}>0$, because $u>0$ and $i$ belongs to $g$.

It is easy to see that the constant $x_{0}$ is equal to

$$
x_{0}=\tan (\pi / 2-a)=\operatorname{cotan} a,
$$

because the Poincaré metric is conformal with respect to the Euclidean metric.

The number $x_{0}$ also satisfies that

$$
u^{2}-2 x_{0} u+1=0 .
$$

Therefore, (2), (3) and (4) give

$$
\tan a=\frac{1}{x_{0}}=\frac{\sqrt{2 \sqrt{2} \cosh t-3}}{\sqrt{2} \cosh t-1}
$$

and the lemma is proved.

Using the lemma, we have that

$$
L\left(\partial B(z, t) \cap H_{0}\right) \geq c e^{t / 2},
$$

for all $z$ in $H$ and for all $t \geq t_{0}$, where $t_{0}$ is any number greater than $\log \sqrt{2}$ and $c$ is a constant which depends only on $t_{0}$ (not in $z$ or $t$ ).

Let $p$ be any point of $\mathcal{N}$. We constructed $\mathcal{N}$ by joining four copies of $W_{1}$. Without loss of generality, we can identify $p$ with a point $z$ of $W_{1} \subset H$. There is a part of $\partial B(p, t)$ which corresponds, by this identification, with $\partial B(z, t) \cap H_{0}$. This is true because a point in $W_{1}$ at distance $t$ of $z$ corresponds with a point in $\mathcal{N}$ at distance $t$ of $p$ : the geodesic arc which joins two points of $W_{1}$ is contained in $W_{1}$ ( $W_{1}$ is geodesicaly convex) and the union of the four copies of $W_{1}$ does not decrease distances in each copy. Consequently,

$$
L_{\mathcal{N}}(p, t) \geq c e^{t / 2}
$$

for all $p$ in $\mathcal{N}$ and for all $t \geq t_{0}$, where $t_{0}$ is any number greater than $\log \sqrt{2}$ and $c$ is a constant which depends only on $t_{0}$. 


\section{5. $\mathcal{N}$ does not have a Green's function}

There is an easy way to convince oneself that this is true. The Riemann surface $\mathcal{N}$ has a Green's function if and only if the Brownian motion in $\mathcal{N}$ is transient ([AS, p. 204], [V1]), i.e., a "Brownian walker" has positive probability to escape to infinity. $\mathcal{N}$ was constructed by joining four copies of $W_{1} . W_{1}$ is "approximately" the horocycle $D=\{\operatorname{Im} z \geq 1\}$. If we transform by a Möbius mapping $T$ the upper-half plane $U$ in the unit disk $\Delta, D$ is transformed in a subdisk of $\Delta$ tangent in one point to $\partial \Delta$. Therefore, there are not many points (there is only one!) in $T(D)$ where the Brownian walker can escape to infinity, and consequently the Brownian motion in $\mathcal{N}$ is not transient.

We give now a rigorous proof. Let $K$ be a relatively compact domain in $\mathcal{N}$. We simply have to prove that the extremal length $\lambda(\Gamma)$ of the family of curves $\Gamma$ "joining" $\partial K$ with the Alexandrov-infinity of $\mathcal{N}$ is infinite [AS, p. 229].

We choose, for example, $K$ to be the union of the four sets $W_{1} \cap\{|z| \leq$ $2\}$ (one for each copy of $W_{1}$ ). Let $\Omega_{n}$ be the union of the four sets $W_{1} \cap\{2 \leq|z| \leq n\}$. If $\Gamma_{n}$ is the set of curves in $\Omega_{n}$ which join $\{|z|=2\}$ with $\{|z|=n\}$, the definition of $\lambda(\Gamma)$ gives

$$
\lambda(\Gamma)=\lim _{n \rightarrow \infty} \lambda\left(\Gamma_{n}\right)
$$

It is a well-known fact [AS, p. 225] that

$$
\lambda\left(\Gamma_{n}\right)=\frac{1}{D\left(u_{n}\right)}
$$

where $D\left(u_{n}\right)$ means the Dirichlet integral in the domain $\Omega_{n}$ of the function $u_{n}$ harmonic in $\Omega_{n}$, with boundary values $u_{n}=0$ in $\partial K$ and $u_{n}=1$ in $\partial \Omega_{n} \backslash \partial K$.

Therefore, if $v_{n}$ is another function in $\Omega_{n}$ with the same boundary values that $u_{n}$, the Dirichlet principle gives

$$
\lambda\left(\Gamma_{n}\right) \geq \frac{1}{D\left(v_{n}\right)}
$$


Let $v_{n}$ be the function defined in each $W_{1} \cap\{2 \leq|z| \leq n\}$ as

$$
v_{n}(z)=\frac{\log (|z| / 2)}{\log (n / 2)} .
$$

We can compute $D\left(v_{n}\right)$ in local coordinates because the Dirichlet integral is a conformal invariant. Then

$$
\begin{aligned}
D\left(v_{n}\right)=4 \iint_{W_{1} \cap\{2 \leq|z| \leq n\}}\left|\nabla v_{n}\right|^{2} d x d y & \leq 4 \int_{0}^{\pi} \int_{2}^{n} \frac{1}{r^{2}} \frac{1}{(\log (n / 2))^{2}} r d r d \theta, \\
D\left(v_{n}\right) & \leq \frac{4 \pi}{\log (n / 2)},
\end{aligned}
$$

and

$$
\lambda\left(\Gamma_{n}\right) \geq \frac{1}{4 \pi} \log (n / 2) .
$$

This proves that $\lambda(\Gamma)=\infty$, and consequently $\mathcal{N}$ does not have a Green's function.

\section{References}

[A] Ahlfors L. V., Sur le type d'une, surface de Riemann, C. R. Acad. Sci. Paris 201 (1935), 30-32.

[AS] Ahlfors L. V. AND Sario L., "Riemann Surfaces," Princeton University Press, Princeton, 1960.

[B] Beardon A. F., "The Geometry of Discrete Groups," SpringerVerlag, New York, 1983.

[Bu] Buser P., A note on the isoperimetric constant, Ann. Sci. Ecole Norm. Sup. (4)15 (1982), 213-230.

[C] Chavel I., "Eigenvalues in Riemannian geometry," Academic Press, Orlando, 1984.

[Ch] Cheeger J., A lower bound for the smallest eigenvalue of the Laplacian, in "Problems in Analysis," Princeton University Press, Princeton, 1970, 195-199.

[D] Doyle P. G., Random Walk on the Speiser graph of a Riemann surface, Bull. Amer. Math. Soc. (2)11 (1984), 371-377.

[E] Epstein C. L., Positive Harmonic Functions on Abelian Covers, Journal of Functional Analysis 82 (1989), 303-315.

[Fe] FenCheL W., "Elementary geometry in hyperbolic space," Walter de Gruyter, Berlin, 1989. 
[F] Fernández J. L., On the existence of Green's function in Riemannian manifolds, Proc. Amer. Math. Soc. 96 (1986), 284-286.

[FR1] FERnÁndez J. L. AND RodRíguez J. M., The exponent of convergence of Riemann surfaces. Bass Riemann surfaces, Ann. Acad. Sci. Fenn. Series A. I. 15 (1990), 165-183.

[FR2] Fernández J. L. And Rodríguez J. M., Area growth and Green's function of Riemann surfaces, Ann. Acad. Scient. Fennica 7 (1982), 249-258.

[G] GRIGOR'YAN A. A., On Liouville theorems for harmonic functions with finite Dirichlet integral, Math. USSR Sbornik 60 (1988), 485-504.

[K] KANAI M., Rough isometries and combinatorial approximations of geometries of non-compact Riemannian manifolds, J. Math. Soc. Japan 37 (1985), 391-413.

[Ka] KARP L., Subharmonic functions, harmonic mappings and isometric immersions, Seminar on Differential Geometry, Ed. S.-T. Yau, Annals of Mathematics Studies, Princeton U. P., 1982.

[L] LYONS T., Instability of the Liouville property for quasi-isometric Riemannian manifolds and reversible Markov chains, J. Diff. Geom. 26 (1987), 33-66.

[LS] Lyons T. AND Sullivan D., Function theory, Random paths and covering spaces, J. Diff. Geom. 19 (1984), 299-323.

[N] Nicholls P. J., Fundamental regions and the type problem for a Riemann surface, Math. Z. 174 (1980), 187-196.

[R] Randol B., Cylinders in Riemann surfaces, Comment. Math. Helv. 54 (1979), 1-5.

[Ro] RodRíGuez J. M., Isoperimetric inequalities and Dirichlet functions of Riemann surfaces, Publicacions Matemàtiques UAB $\mathbf{3 8}$ (1994), 243-253.

[SN] SaRio L. AND NAKAI M., "Classification theory of Riemann surfaces," Springer-Verlag, Berlin, 1970.

[S] Sullivan D., Related aspects of positivity in Riemannian geometry, J. Diff. Geom. 25 (1987), 327-351.

[T] TsujI M., "Potential theory in modern function theory," Maruzen, Tokyo, 1959.

[V1] Varopoulos N. Th., Potential theory and diffusion on Riemannian manifolds, Conference in Harmonic Analysis in honor of Anthony Zygmund, Wadsworth, Belmont, California, 1983.

[V2] Varopoulos N. Th., Small time Gaussian estimates of heat 
diffusion kernels. Part I: The semigroup technique, Bull. Sc. Math. 113 (1989), 253-277.

[V3] VAROPOUlos N. TH., Information theory and harmonic functions, Bull Sc. Math. 110 (1986), 347-389.

\author{
Universidad Carlos III de Madrid \\ Escuela Técnica Superior \\ Departamento de Ingeniería \\ C/ Butarque 15 \\ 28911 Leganés \\ MADRID
}

Rebut el 19 de Setembre de 1994 